\title{
BMJ Open Can children swallow tablets? Outcome data from a feasibility study to assess the acceptability of different-sized placebo tablets in children (creating acceptable tablets (CAT))
}

To cite: Bracken $L$, McDonough E, Ashleigh S, et al. Can children swallow tablets? Outcome data from a feasibility study to assess the acceptability of different-sized placebo tablets in children (creating acceptable tablets (CAT)). BMJ Open 2020;10:e036508. doi:10.1136/ bmjopen-2019-036508

- Prepublication history for this paper is available online. To view these files, please visit the journal online (http://dx.doi. org/10.1136/bmjopen-2019036508).

Received 17 December 2019

Revised 11 June 2020

Accepted 06 August 2020

Check for updates

(C) Author(s) (or their employer(s)) 2020. Re-use permitted under CC BY-NC. No commercial re-use. See rights and permissions. Published by BMJ.

For numbered affiliations see end of article.

Correspondence to

Professor Matthew Peak;

Matthew.Peak@alderhey.nhs.uk

\section{ABSTRACT}

Objective Feasibility study to investigate the acceptability of different-sized placebo tablets in children aged 4-12 years.

Design and setting Clinical Research Facilities, inpatient wards and outpatient clinics within a Regional Paediatric Hospital and/or District General Hospital. Healthy children and National Health Service (NHS) patients were asked to swallow three placebo tablets: $6 \mathrm{~mm}, 8 \mathrm{~mm}$ and $10 \mathrm{~mm}$, smallest to largest. The researcher observed children's facial expressions and behaviours on swallowing and measured the volume of water consumed. Participants completed a questionnaire about the overall acceptability; including swallowability, taste and volume of water consumed. For analysis, participants were stratified by age: 4-8 years and 9-12 years.

Results The feasibility study led to an estimated recruitment rate of $0.8 \%$ for NHS inpatients and 211 healthy children over a 1-year period. In total, 55 participants were recruited, 30 to the younger group, of which $77 \%$ had never taken a tablet before. $84 \%$ of the 25 older children had previously taken a tablet. All participants attempted to swallow the smallest sized tablet. The children aged 4-8 years found the larger tablets easier to swallow, however the older children found little difference between the tablet sizes. The younger children required more water to swallow each tablet size compared with the older children where an increasing volume of water was consumed as tablet size increased. Taste was rated highly for both age groups. The $8 \mathrm{~mm}$ tablets were deemed the most acceptable tablet size by all participants.

Conclusion Tablets are potentially an acceptable formulation for children aged 4-12 years. Most children aged 4-8years who attempted to swallow tablets successfully did so. Recruitment of NHS inpatients to medicine acceptability studies is challenging, however, recruitment of children of staff proved an effective strategy. Valuable lessons have been learnt from this feasibility study which will inform the design of a larger definitive trial.

\section{INTRODUCTION}

The development of age-appropriate medicines acceptable to the paediatric population

\section{Strengths and limitations of this study}

- This study was conducted in both inpatients and healthy children to determine the overall acceptability (which includes swallowability, volume of water consumed and taste) of tablets in both children who have never taken tablets before and children who have experience in taking tablets.

- A direct comparison of each tablet size in each child was drawn based on both researcher observations and participant questionnaires.

- The order in which tablets were attempted and/ or swallowed was not randomised in this study. Participants took them in size order from smallest to largest due to safety concerns. However, future studies could include randomisation as no safety issues were observed.

- The sample size in this study is relatively small at 55 participants for definitive conclusions to be drawn.

- Only five children aged 4 years and two children aged 5 years participated in the study, therefore in order for more reliable conclusions to be drawn in younger children, more children aged 4-5 years would need to be recruited.

is an area of great interest. The number of medicines authorised for use in children has increased over the last 40 years, although ensuring medicines are age-appropriate has only gradually increased more recently. ${ }^{1}$ It is evident that there are insufficient ageappropriate medicines for children of all ages and ensuring medicines are age appropriate and acceptable to patients is vital for both drug efficacy and safety. ${ }^{1}$

The European Medicines Agency defines patient acceptability as 'the overall ability and willingness of the patient to use and its caregiver to administer the medicine as intended'. ${ }^{2}$ Its 'Guideline on Pharmaceutical 
Development of Medicines for Paediatric Use' recognises that acceptability is likely to impact on patients' adherence to a medication and therefore assessment of medicine acceptability within the paediatric population should be an essential part of pharmaceutical and clinical development of medicines. ${ }^{2}$ In addition, the acceptability of a medicine should preferably be studied in children themselves. ${ }^{2}$ Therefore, studying acceptability of medicines in the paediatric population is essential.

Acceptability of paediatric dosage forms is known to differ between children, and this is influenced by several factors including age, behaviour, background and disabilities. $^{2}$ There have been many studies conducted on the acceptability of paediatric medicines but there are still large gaps in the knowledge base on this area. ${ }^{3}$ In addition, there is a lack of consensus on the methods for medicine-acceptability assessment. ${ }^{4}$ The majority of studies have used a Visual Analogue Scale or a hedonic scale. ${ }^{3}$ Liquid medicines have long been considered the most appropriate oral dosage form for children and young people (CYP); however, support is increasing for solid dosage forms to be used more frequently due to their better stability profile, suitability for coating to taste mask and the inclusion of a lower number of excipients. ${ }^{5}$ There is increasing evidence to show that tablets are an acceptable dosage form and a suitable alternative to liquids which can overcome the challenges associated with liquid medicines. ${ }^{6-9}$

The ability of CYP to swallow tablets is of importance to the pharmaceutical sector, healthcare professionals and patients themselves. The regulatory drive to develop ageappropriate formulations for children is hampered by the lack of empirical knowledge of the optimal tablet size for children of different ages. As a consequence, pharmaceutical formulators and manufacturers may be producing medicines for children in age-inappropriate tablet sizes, primarily because of the swallowability of different tablet sizes. Intersector consortia such as the European Paediatric Formulations Initiative (EuPFI) have highlighted the importance of a better understanding of children's ability to swallow different-sized tablets. ${ }^{10}$ This is best achieved through studies using direct administration of tablets to children. In 2016 a paediatric formulation development workshop organised by a collaborative partnership between the University of Maryland, the International Consortium for Innovation and Quality in Pharmaceutical Development (IQ consortium) and the EuPFI, held sessions on swallowability and palatability assessments and summarised their findings in a report, highlighting the need for further studies. ${ }^{11}$

A definitive trial comparing the acceptability of different-sized tablets in children is not possible because there are no available outcome data to compute a sample size estimate and the feasibility of such a study has not been explored. Therefore, a feasibility study was designed based on best practice guidance ${ }^{12}$ to obtain estimates of key parameters needed to design a definitive trial of the acceptability of different-sized placebo tablets by children.
Key feasibility parameters included recruitment rate, consent rate, compliance with interventions and acquisition of outcome data. In addition, we aimed to generate estimates of measures of acceptability including: swallowability, taste and volume of water consumed to inform a sample size estimate for a future definitive trial.

\section{METHODS}

\section{Study design}

A feasibility study to investigate the overall acceptability of different-sized placebo tablets in children. Acceptability includes swallowability, taste and the volume of water consumed to swallow each tablet.

\section{Setting}

The study took place on the National Institute for Health Research Clinical Research Facilities and inpatient wards within a Regional Paediatric Hospital and a District General Hospital (DGH), in addition to outpatient clinics within the Regional Paediatric Hospital.

\section{Participants}

Children aged 4-12 years, able to read and/or understand English with no known difficulty swallowing and no allergy to peanuts, gluten and/or lactose. Both patients (children under the care of a Regional Paediatric Hospital or DGH) and healthy volunteers were included in the study. A healthy volunteer was defined as a participant who was not an inpatient or an outpatient attending a hospital appointment on the day of participation.

\section{Patient and public involvement}

CYP were involved throughout the concept, design and delivery of the study. Prioritisation of research question: the acceptability of tablets, particularly size, has been reported as a concern by CYP to this research group in workshops and individual encounters. Design of study information materials and introductory video: these were reviewed by the Liverpool Young Persons' Advisory Group (YPAG) and written materials modified based on its feedback. The study design was presented to the Liverpool YPAG and specific methods were adapted in response to feedback. This included: the order of tablets to be administered from smallest to largest; the decision not to randomise the tablet administration sequence and to ask a question about this in the context of a future trial.

\section{Identification and consent}

National Health Service (NHS) patients were screened by age, and where children within the correct age range were identified, the research team then approached the clinical team to check their eligibility in more depth before approaching the family to tell them about the study. An age-appropriate participant information sheet was given to the patient, and a detailed parent information sheet was given to the parent/legal guardian. They were given adequate time to read the information provided and for the opportunity to ask questions. Informed consent was 
obtained from the parent/legal guardian and written assent was obtained for children aged 6 and over who wished to participate.

Following informed consent, participants were shown a short video of a 3-year-old child demonstrating how to swallow a tablet, before being presented with the smallest of the three tablets.

\section{Interventions}

Placebo tablets $(6 \mathrm{~mm}, 8 \mathrm{~mm}$ and $10 \mathrm{~mm}$ diameter with a standard round biconvex shape) were manufactured to Good Manufacturing Practice standards by Quotient Sciences. Tablets contained microcrystalline cellulose coated with an OPADRY clear coating which did not contain a flavour. Participants were given the tablets in a standardised order, from smallest to largest, each receiving a single-dose exposure. Each participant received $150 \mathrm{~mL}$ of water with each tablet and participants had free access to additional water to swallow each tablet, if required. Tablets were presented to the children in medicine pots and the children then self-administered the medication. A short ( $5 \mathrm{~min}$ ) break was offered to participants between each tablet to minimise participants' discomfort. They were given the opportunity to withdraw from the study after attempting to take each tablet; and even if unsuccessful at that size, they could continue on with the study if they wished to do so.

\section{Staff training}

Prior to commencing the study, all research staff members were trained by PM. This involved watching a training video which showed the various facial expressions and behaviours to be aware of. Staff then completed an assessment of facial expressions which was compared to confirm their understanding and check for the accuracy of detecting the different expressions. This ensured standardisation of the results captured as part of the researcher observations.

\section{Screening data}

An additional aim of the study was to use screening logs to estimate recruitment rates of children attending hospital to an acceptability study. Screening data for all wards in the regional paediatric hospital, with the exception of the Oncology, High Dependency Unit (HDU) and Paediatric Intensive Care Unit (PICU), were obtained over a 4-week period: 2 weeks during the summer season and 2 weeks during the winter season. This was obtained as a measure of the feasibility to recruit inpatients to acceptability studies, and the data were extrapolated to calculate the feasibility of recruiting inpatients over a 12-month period.

The feasibility of recruiting healthy children was also calculated. Active recruitment for healthy children took place over a 57-day period, and via extrapolation, it was possible to estimate recruitment over a 12-month period.
A

Facial expressions observed

\begin{tabular}{|l|l|}
\hline Expression & Tick if observed \\
\hline Brow bulge/lower (frown) & \\
\hline Eyes squeezed shut or towards shut & \\
\hline Nose wrinkle & \\
\hline Pursed lips & \\
\hline
\end{tabular}

B

Behaviours observed

\begin{tabular}{|l|l|}
\hline During/prior to administration & Tick if observed \\
\hline Child voices resistance & \\
\hline Child cries & \\
\hline Child refuses test sample & \\
\hline Immediately after administration & \\
\hline Child voices disgust & \\
\hline Child vomits & \\
\hline Child spits out test sample & \\
\hline Child cries & \\
\hline Child chews sample & \\
\hline
\end{tabular}

C

Hedonic Scale
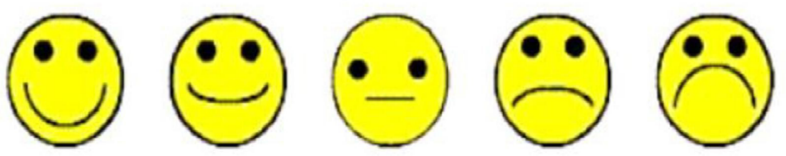

Very Easy

Very Hard

Figure 1 Researcher observations and participant questionnaires were completed after each tablet was attempted. (A, B) are taken from the Researcher Observation Sheet and show the facial expressions and behaviours recorded for each participant. (C) Shows the hedonic scale on which participants were asked to rate four characteristics of each tablet.

\section{Outcomes}

Assessment of swallowability

Using the Researcher Observation Sheet (figure 1A,B), a researcher observed the participant attempting to take each tablet. Their observations were recorded immediately after the attempt to swallow each tablet was made along with any verbal comments given regarding the swallowability of each tablet. Participants also completed a short paper questionnaire immediately after each attempt, and a maximum of three questionnaires were completed per participant. Tablets were evaluated by 
participants using 5-point hedonic scales (figure 1C) to obtain their views on the swallowability of the tablet. The term swallowability was explained to children using ageappropriate language. Numerical values were assigned to the hedonic scales from 1 (very easy) to 5 (very hard) for data analysis. Values of 1-3 on the hedonic scale were deemed acceptable.

The total number of participants in each group who attempted and successfully swallowed each size tablet was recorded. Each participant was asked whether they had any previous experience of swallowing tablets.

\section{Assessment of the volume of water required to swallow the tablet}

The volume of water consumed by each participant was determined by the researcher measuring the volume of water remaining in the cup after a participant had taken each tablet. The participant was also asked to rate the volume of water required with each tablet administration, on a hedonic scale (figure 1C) using the anchors "very good' to 'very bad' when asked 'what did you think of the amount of water you had to drink?'. Numerical values of 1-3 on the hedonic scale were deemed acceptable.

\section{Assessment of taste}

Although each sized tablet in theory should have tasted the same (being coated with an identical film coating system), each participant was asked to rate the taste of the tablet swallowed using the hedonic scale as part of the questionnaire. This was to assess whether participants noticed any difference with the increasing tablet size. The anchors used were 'very good', 'very bad' on the hedonic scale and numerical values of 1-3 were deemed acceptable.

\section{Assessment of overall acceptability}

Participants were asked to rate how acceptable the tablet was to take directly following each administration using a hedonic scale (figure 1C). Anchors used were "very good' to 'very bad' and a score of 1-3 was deemed acceptable. Facial expressions and behaviours prior to, during and after administration of each tablet were observed and recorded by a researcher using the tick box chart displayed in figure 1A,B. The sum (0-4) of the negative facial expressions (figure 1A) exhibited by each participant was calculated as a measure of their discomfort or dislike of the tablet: a value of 0 was given if there were no negative facial expressions observed; 1-indicated light discomfort; 2-moderate discomfort; 3-considerable discomfort; 4-severe discomfort. A total score was calculated based on any facial expressions and/or behaviours observed by the researcher at the time prior to, during or following each tablet administration attempt. Any additional verbal comments from the participant in terms of acceptability were also noted.

Participants were asked if they would be willing to take each tablet every day, if it were a medicine. They were also asked how often they currently take medicines and what would be the most important factor if they had to take

\begin{tabular}{|c|c|c|}
\hline Week & Screened & New admissions \\
\hline Winter week 1 & 256 & 155 \\
\hline Winter week 2 & 260 & 101 \\
\hline Summer week 1 & 300 & 110 \\
\hline Summer week 2 & 261 & 108 \\
\hline Total & 1077 & 474 \\
\hline
\end{tabular}

medicines every day, from a list of options: taste, smell, tablet size, taste left in the mouth or texture.

\section{RESULTS}

\section{Screening data}

Inpatients

The demographic of the inpatient population of the regional paediatric hospital was reviewed during the 4-week screening period. There were 1077 patients aged 4-12 years across six wards (all wards with the exception of HDU/PICU and oncology). These patients were screened across the four screening weeks and represent 474 individual patients (new admissions) in the correct age range (table 1$)$.

In parallel to the top-level screening (where patients were only screened by age range) of the six wards, a general medical ward was prospectively screened for study participant eligibility by age to determine which inpatients were eligible; who could subsequently be approached; and how many eligible inpatients would agree to participate in the study. These data are summarised in figure 2.

A total of 474 individual inpatients in the typical NHS inpatient setting within the regional paediatric hospital over a 4-week period met the age criteria for the study. Using this figure, an estimate for the maximum number of eligible children (by age) over the period of a year could be calculated: 6162 eligible participants (no. of eligible participants by age over a 4 -week period/4*52) for the study. Screening of the general medical ward allowed calculation of a recruitment yield, $2 / 250=0.008$, which represents $0.8 \%$. Recruitment of only $0.8 \%$ of eligible participants would result in an annual theoretical maximum of the recruitment of 49 inpatients across Alder Hey Children's NHS Foundation Trust, a typical NHS hospital setting for children. This demonstrates that a definitive trial is not feasible through recruitment of inpatients in this setting. The consent rate of eligible participants was low 2/39 (5\%); some of the reasons recorded for eligible participants who refused to participate included: patient refusal, infection, health issues/ disability, safeguarding issue, patients discharged and patient away from the ward. Despite recruitment being challenging, once participants were recruited to the study compliance with the protocol was good. 


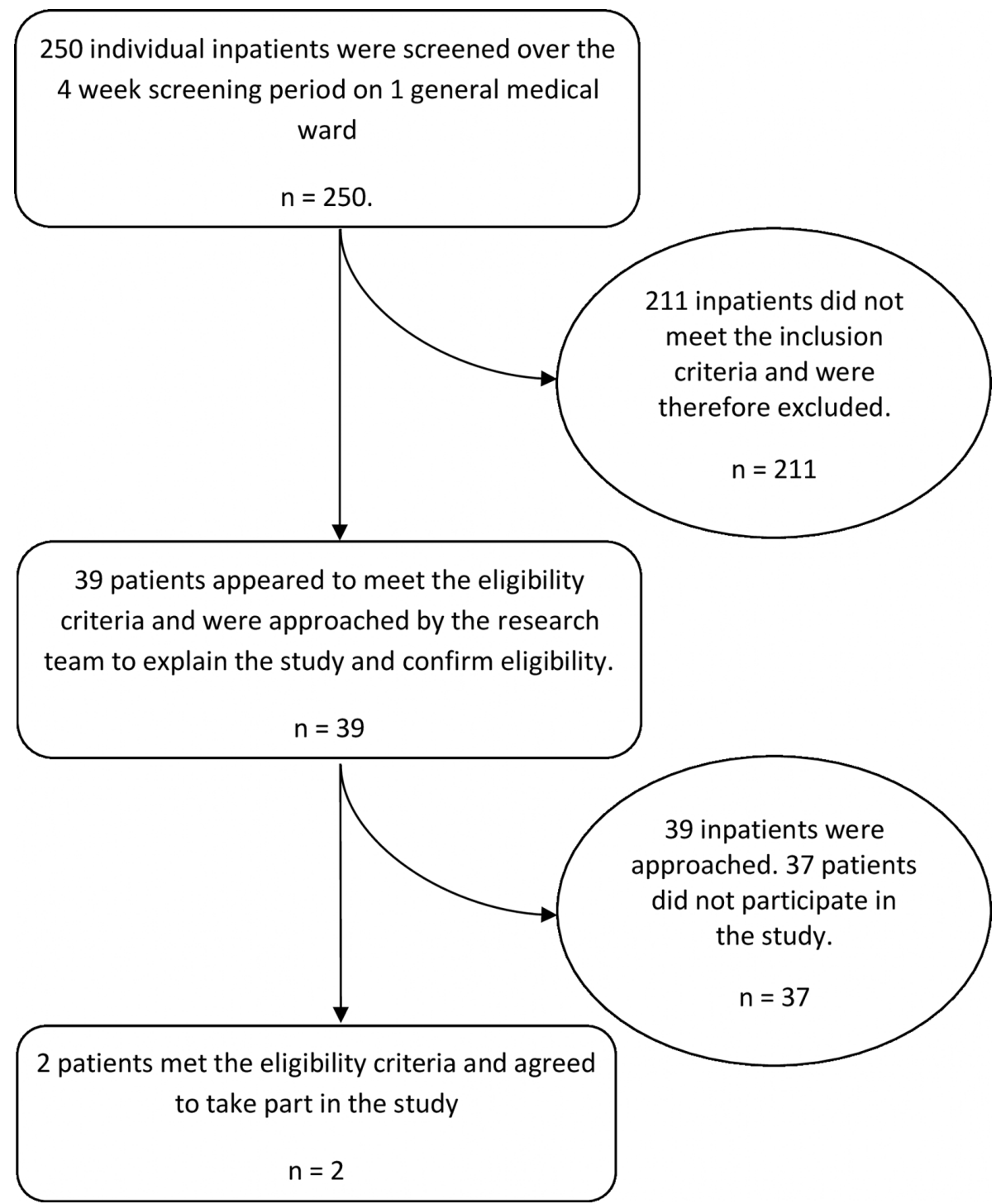

Figure 2 Recruitment flow chart.

\section{Healthy children}

The feasibility of recruiting healthy children was also estimated during this study. Posters were placed around the hospital, an advertisement was placed on the staff intranet and word of mouth was used to promote the study. Healthy children were recruited both within a Regional Paediatric Hospital and a DGH. A total of 33 healthy children were recruited to the study over a period of 57 days of active recruitment.

Using this figure, an estimate for the number of eligible healthy children over the period of a year can be calculated. If 33 children were recruited over a period of 57 days, there is the possibility to recruit 211 healthy children over a 1-year period.

\section{Demographics}

A total of 55 participants (33 healthy volunteers and 22 NHS patients) (table 2) were recruited to the study, all aged between 4 and 12 years (median age $=8$, mean age $=8.1)$. For analysis, the participants were stratified by age into two groups: $4-8$ years (median age $=6$, mean age $=6.3$ ) and $9-12$ years (median age $=10$, mean age $=10.2)$. Thirty participants $(54.5 \%$ of total participants) were in the younger age group and 25 were in the older age group. Twenty-three per cent of participants aged 4-8years and $84 \%$ of participants aged 9-12years had previously taken a tablet before participating in this study. The majority $(77 \%)$ of participants aged 4-8years were tablet naive.

\section{Tablet swallowability}

Researcher observations of negative facial expressions

In 50\% of participants aged 4-8 years and $16 \%$ of participants aged 9-12 years, at least one negative facial expression was observed prior to or during administration of the $6 \mathrm{~mm}$ tablet. The most commonly observed $(30 \%)$ facial expression in the 4-8-year-old group was 'brow bulge', and this was followed by 'eyes squeezed' and 'nose wrinkle' which were both demonstrated by $13 \%$ of participants, and finally 'pursed lips' exhibited by $10 \%$. In the 9-12year-old group, the most commonly observed $(12 \%)$ facial expression was 'eyes squeezed', and this was 


\begin{tabular}{lrr} 
Table 2 Participant demographics & & \\
\hline & Frequency (n) & $\%$ \\
\hline Gender & 26 & 47.3 \\
$\quad$ Male & 29 & 52.7 \\
$\quad$ Female & & \\
Age (years) & 30 & 54.5 \\
4-8 & 25 & 45.5 \\
9-12 & & \\
Taken a tablet previously? & 28 & 50.9 \\
Yes & 27 & 49.1 \\
No & & \\
How often do you take medicines? & 11 & 20.0 \\
Every day & 1 & 1.8 \\
Every week and when poorly & 40 & 72.7 \\
When poorly & 2 & 3.6 \\
Never & 1 & 1.8 \\
No response & & \\
\hline
\end{tabular}

followed by 'pursed lips' (8\%), and 'brow bulge' and 'nose wrinkle' (both 4\%).

In $52 \%$ of participants aged $4-8$ years and $28 \%$ of participants aged 9-12 years, at least one negative facial expression was demonstrated prior to or during administration of the $8 \mathrm{~mm}$ tablet. The most commonly observed (35\%) facial expression in the 4-8-year-old group was 'brow bulge', and this was followed by 'eyes squeezed and 'nose wrinkle' which were both demonstrated by $17 \%$ of participants, and finally 'pursed lips' exhibited by $4 \%$. In the 9-12 year-old group, the most commonly observed (16\%) facial expression was 'eyes squeezed' and this was followed by 'nose wrinkle' and 'pursed lips' (both $8 \%$ ) and 'brow bulge' $(4 \%)$.

In $48 \%$ of participants aged $4-8$ years and $24 \%$ of participants aged 9-12 years, at least one negative facial expression was observed prior to or during administration of the $10 \mathrm{~mm}$ tablet. The most commonly observed (24\%) facial expressions in the 4-8-year-old group were 'brow bulge' and 'nose wrinkle', and these were followed by 'eyes squeezed' (19\%) and 'pursed lips' (5\%). Of those in the 9-12-year-old group, the most commonly observed (12\%) negative facial expression was 'pursed lips' and this was followed by 'brow bulge' and 'eyes squeezed' (both 8\%). Nobody in the 9-12-year-old group displayed 'nose wrinkle' when attempting to take the $10 \mathrm{~mm}$ tablet. The results are summarised in table 3 .

\section{Successful swallowing}

$6 \mathrm{~mm}$ tablets

For the whole study population, $45 / 55(81.8 \%)$ of participants were able to successfully swallow (no residue

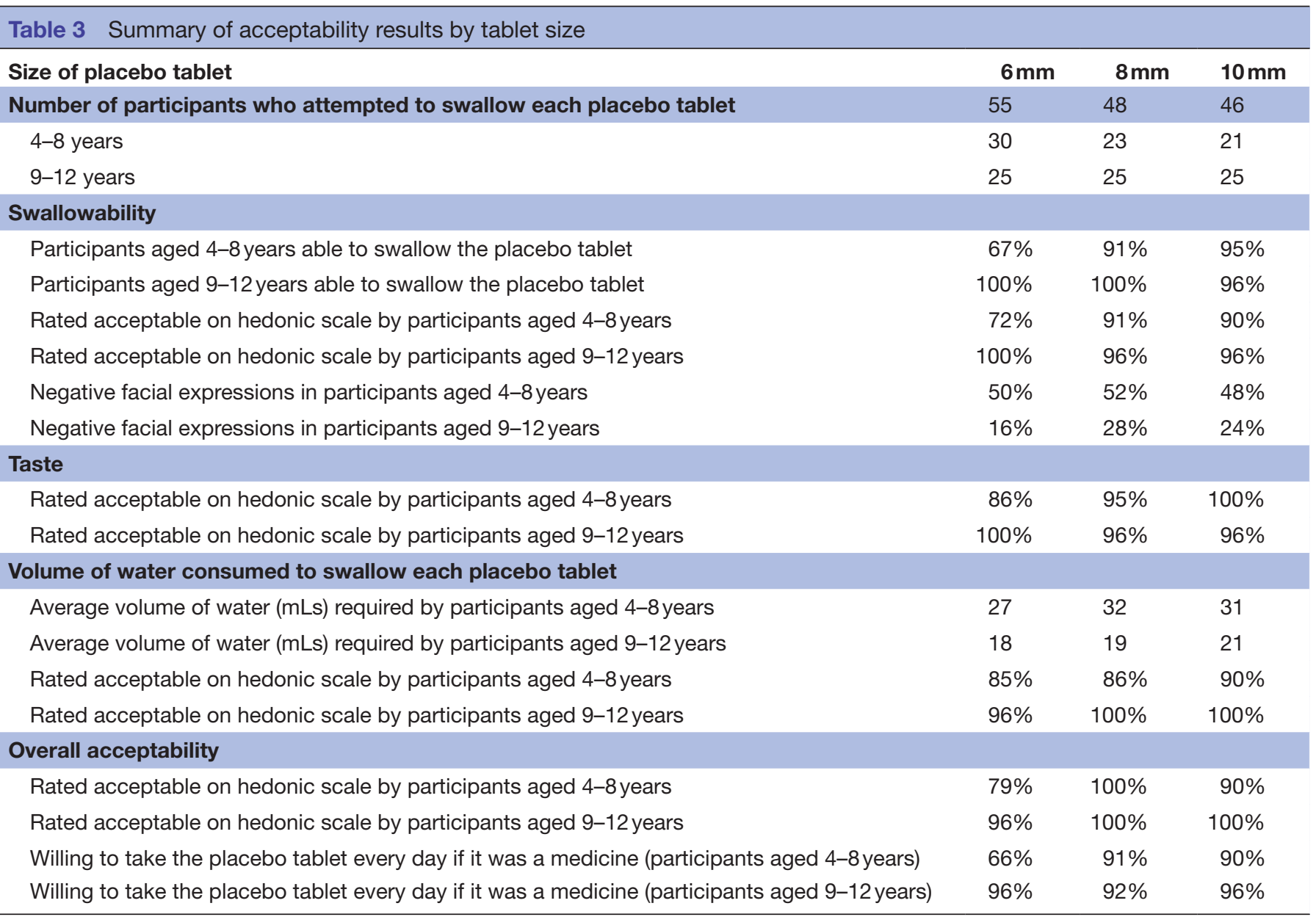




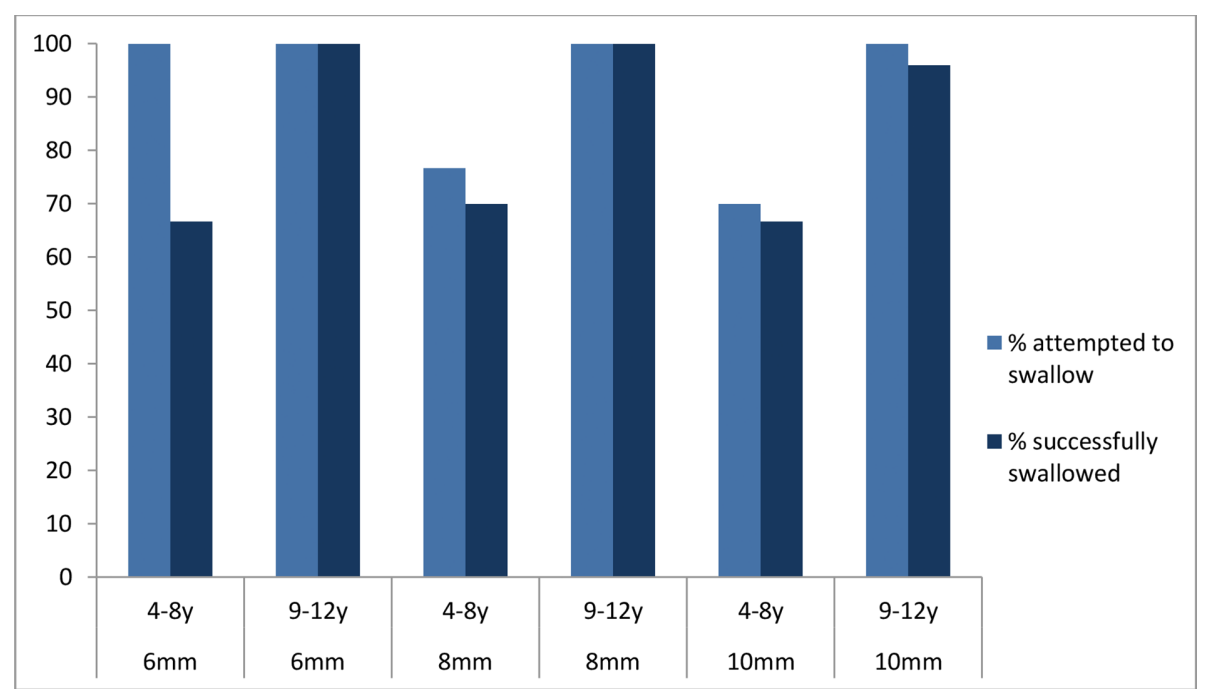

Figure 3 Proportion of participants aged 4-8 years and 9-12 years who attempted to swallow and successfully swallowed each tablet size.

remaining in child's mouth on inspection) a $6 \mathrm{~mm}$ placebo tablet: $67 \%$ of participants aged $4-8$ years and $100 \%$ of participants aged 9-12years successfully swallowed the $6 \mathrm{~mm}$ tablet (figure 3 ). In the 4-8-year-old group, there was an association $(n=5)$ between increase in age and the ability to successfully swallow a $6 \mathrm{~mm}$ tablet (proportion of different-aged participants able to swallow a $6 \mathrm{~mm}$ tablet: 4 years-20\%; 5 years-50\%; 6 years-78\%; 7 years- $83 \%, 8$ years- $75 \%$ ). Of the 10 children who were unable to swallow the $6 \mathrm{~mm}$ tablet, $80 \%$ had never taken a tablet before. Three participants who were unable to swallow the $6 \mathrm{~mm}$ tablet managed to successfully swallow the $8 \mathrm{~mm}$ and $10 \mathrm{~mm}$ tablets. Two of these individuals were age 6 and the other was age 8 . The 8 -year-old participant asked to attempt to swallow the $6 \mathrm{~mm}$ tablet again after taking the $8 \mathrm{~mm}$ and $10 \mathrm{~mm}$ tablets and succeeded.

\section{$8 \mathrm{~mm}$ tablets}

Seven of $30(23 \%)$ participants aged $4-8$ years did not attempt to swallow the $8 \mathrm{~mm}$ tablet, and $71 \%$ of these individuals had never taken a tablet before this study. Ninety-one per cent of participants aged 4-8years and $100 \%$ of participants aged 9-12years who attempted to take the $8 \mathrm{~mm}$ tablet were successful. The two children who were unable to swallow the $8 \mathrm{~mm}$ tablet were 5 and 7 years old, respectively.

\section{$10 \mathrm{~mm}$ tablets}

Forty-six (84\%) of the 55 participants attempted to swallow the $10 \mathrm{~mm}$ tablet and for the overall study population, $96 \%$ of those who attempted to swallow the $10 \mathrm{~mm}$ tablet, succeeded in doing so. Ninety-five per cent of participants aged 4-8years and 96\% of participants aged 9-12years who attempted to take the $10 \mathrm{~mm}$ tablet successfully swallowed it.

Overall, these data suggest that for the older age group studied (9-12 years of age), there is no association between tablet size and swallowability as all three sizes of tablet were swallowed by between $96 \%$ and $100 \%$ of participants, However, in the younger age group (4-8 years of age), swallowability appeared to be inversely associated with tablet size as only $67 \%$ were able to swallow the $6 \mathrm{~mm}$ tablet compared with $91 \%(8 \mathrm{~mm})$ and $95 \%(10$ $\mathrm{mm}$ ) for the larger sized tablets.

\section{Volume of water consumed}

In the 4-8-year-old group the average water consumption required to swallow each tablet size was: $6 \mathrm{~mm}-27 \mathrm{~mL}$; $8 \mathrm{~mm}-32 \mathrm{~mL} ; 10 \mathrm{~mm}-31 \mathrm{~mL}$. In the 9-12-year-old group the average water consumption required to swallow each tablet size was: $6 \mathrm{~mm}-18 \mathrm{~mL} ; 8 \mathrm{~mm}-19 \mathrm{~mL} ; 10 \mathrm{~mm}-21 \mathrm{~mL}$. Therefore, the younger age group required more water ( $10.3 \mathrm{~mL}$ on average) to swallow each tablet size. In the 9-12-year-old age group there is a trend of increasing tablet size and increasing volume of water consumed of water, $1.0-2.1 \mathrm{~mL}$ extra water consumed as tablet size increased.

For all sized tablets, very little difference was reported across the age groups for the acceptability of the volume of water required to be consumed. Thus, $85 \%-96 \%$ of participants aged $4-8$ years and $96 \%-100 \%$ of participants aged 9-12years rated this as acceptable or better using the hedonic scale (table 3 ).

\section{Taste}

From the participant reports on tablet taste, participants aged $4-8$ years rated the $6 \mathrm{~mm}$ tablet as $86 \%, 8 \mathrm{~mm}$ as $95 \%$ and $10 \mathrm{~mm}$ as $100 \%$ acceptable or better. The participants aged 9-12 years rated the $6 \mathrm{~mm}$ as $100 \%$, whereas both the $8 \mathrm{~mm}$ and $10 \mathrm{~mm}$ were rated as $96 \%$ acceptable or better (table 3).

\section{Overall acceptability}

As shown in table 3 , the $8 \mathrm{~mm}$ tablets were rated to be the most acceptable tablet size by all participants across both age groups. 


\section{$6 \mathrm{~mm}$ tablets}

The most positively rated attribute of the $6 \mathrm{~mm}$ tablet in the 4-8-year-old group was taste, which was deemed acceptable by $86 \%$ of this group, and this was followed by volume of water consumed $(85 \%)$, acceptability $(79 \%)$ and swallowability $(72 \%)$.

In the 9-12-year-old group, the most positively rated attributes were taste and swallowability, with $100 \%$ of this group rating these attributes as acceptable or better, followed by volume of water consumed and acceptability which were rated as acceptable or better by $96 \%$ of participants aged 9-12years.

\section{$8 \mathrm{~mm}$ tablets}

The most positively rated attribute of the $8 \mathrm{~mm}$ tablet in the 4-8-year-old group was acceptability, gaining a positive score (1, 2 or 3 on the hedonic scale) from $100 \%$ of participants in this group. This was followed by taste which was deemed acceptable or better by $95 \%$ of participants aged 4-8 years, swallowability (91\%) and volume of water consumed $(86 \%)$.

In the 9-12-year-old group, the most positively rated attributes were volume of water consumed and acceptability, which were rated as acceptable or better by $100 \%$ of participants in the 9-12-year-old group. Both swallowability and taste were rated as acceptable or better by $96 \%$ of participants aged 9-12years.

\section{$10 \mathrm{~mm}$ tablets}

The most positively rated attribute of the $10 \mathrm{~mm}$ tablet in the 4-8-year-old group was taste which was rated as acceptable or better by $100 \%$ of participants aged $4-8$ years. This was followed by swallowability, volume of water consumed and acceptability (all 90\%).

In the 9-12year age group, the most positively rated attributes of the $10 \mathrm{~mm}$ tablet were volume of water consumed and acceptability, rated as acceptable or better by $100 \%$ of participants in the $9-12$-year-old group. Both swallowability and taste were rated as acceptable or better by $96 \%$ of participants aged $9-12$ years.

Willingness to take the tablet every day if it were a medicine Overall, responses indicated that $80 \%$ of participants (66\% of participants aged 4-8 years and $96 \%$ of participants aged 9-12 years) would be willing to take the $6 \mathrm{~mm}$ tablet size every day if it were a medicine, compared with $91 \%$ of participants $(91 \%$ of participants aged $6-8$ years and $92 \%$ of participants aged 9-12 years) for $8 \mathrm{~mm}$ and $93 \%$ of participants $(90 \%$ of participants aged $6-8$ years and $96 \%$ of participants aged 9-12 years) for $10 \mathrm{~mm}$.

\section{Most important factor if you had to take tablets as a medicine} All participants were asked what they thought the most important factor was if they had to take tablets as a medicine. The most common response from both age groups was tablet size and this answer accounted for $53 \%$ of responses in the participants aged 4-8years and $50 \%$ of responses in participants aged 9-12years (figure 4), although some participants gave more than one response.

\section{DISCUSSION}

The study is the first attempt in systematically evaluating the ability and acceptance of preschool and school children in swallowing conventional sized tablets. This study demonstrated that tablets of $6 \mathrm{~mm}, 8 \mathrm{~mm}$ and $10 \mathrm{~mm}$ are potentially an acceptable formulation for children aged 4-12 years. Mini-tablets (defined as those less than $5 \mathrm{~mm}$ in diameter ${ }^{13}$ ) were purposefully not evaluated as part of this study, in order to specifically focus on the swallowability of larger sized tablets in children where there are limited data available. Several studies have been published on the swallowability of mini-tablets in the infant and child population. ${ }^{714}$

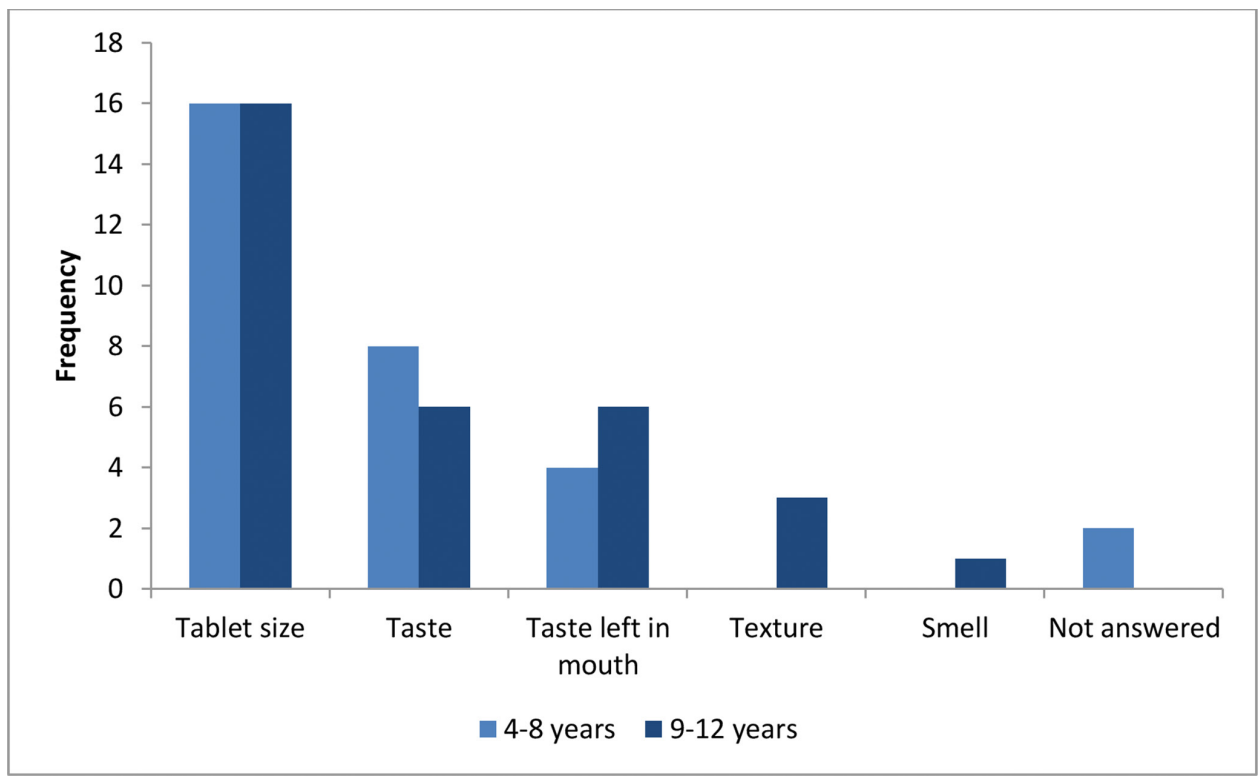

Figure 4 Participant important factors if they had to take tablets as a medicine. 
Nearly all the older children (aged 9-12 years) successfully swallowed all three sized tablets and the majority of the younger children (aged 4-8 years) who attempted to swallow the $6 \mathrm{~mm}, 8 \mathrm{~mm}$ and $10 \mathrm{~mm}$ tablets successfully did so $(67 \%, 91 \%$ and $95 \%$, respectively). In the younger age group, an age-related trend was observed in their ability to swallow the tablets successfully with $20 \%, 50 \%$ and $78 \%$ successfully swallowing the $6 \mathrm{~mm}$ tablets for 4-, 5- and 6-year-old subgroups, respectively. However, due to the small sample size of each age subset $(\mathrm{n}=5,2$ and 9 for the 4-, 5- and 6-year-old groups, respectively), a meaningful conclusion cannot be drawn. Another observation was that the younger children who successfully swallowed the $6 \mathrm{~mm}$ tablets were able to succeed in swallowing the $8 \mathrm{~mm}$ and $10 \mathrm{~mm}$ tablets which was demonstrated in one 4-year-old participant, one 5-year-old participant and seven 6-year-old participants. A previous report in a small 4-9-year-old cohort demonstrated that children were able to gradually increase the pill sizes that they can swallow by training.$^{15}$ A similar finding was demonstrated in this study but again higher numbers of participants of these ages would need to be evaluated to draw any definitive conclusion. Interestingly, three participants in the younger group who failed to swallow the $6 \mathrm{~mm}$ tablet on their first attempt were able to successfully swallow the $8 \mathrm{~mm}$ and $10 \mathrm{~mm}$ tablets. This demonstrates a potential learning effect. Previous literature has also demonstrated this learning effect, with Hansen et al noting that 'repetition and experience was a key' to children successfully swallowing tablets ${ }^{16}$ and pill-swallowing training in children dates back to the $1980 \mathrm{~s}^{17}$

The acceptance of the tablets was demonstrated in both the younger and older age groups with only a small proportion of the younger children refusing the tablets (7\%, $4 \%$ and $0 \%$ for $6 \mathrm{~mm}, 8 \mathrm{~mm}$ and $10 \mathrm{~mm}$ tablets, respectively) and no participants in the 9-12-year-old group refusing any of the tablet sizes. In some instances, the tablets were accepted but were then spat out $(6 \mathrm{~mm}$ : $18 \%, 8 \mathrm{~mm}: 4 \%, 10 \mathrm{~mm}: 4 \%$ ), and all of these instances of spitting out, with the exception of one participant with the $10 \mathrm{~mm}$ tablet, occurred in the 4-8-year-old group. No vomiting or choking was observed or reported in either age group for any tablet size, however researcher observations displayed a level of dislike and discomfort in both age groups as the display of at least one negative facial expression. Despite this, the majority of participants agreed they would be willing to take the tablets every day if they were required to take medicines regularly. Over $80 \%$ of participants reported they would be happy to take the $6 \mathrm{~mm}$ tablet every day, and $91 \%(8 \mathrm{~mm})$ and $93 \%(10 \mathrm{~mm})$ would be willing to take the other tablet sizes based on participant reported outcomes. All participants who reported they would be willing to take a certain size tablet every day if it were a medicine successfully swallowed the corresponding tablet size. Overall, the 9-12-year-old group showed better acceptance of all tablets than the 4-8-year-old group, although this could be attributed to the fact that a larger proportion of the participants aged 4-8years had not encountered taking tablets previously which was therefore a new experience for them. In total, $25 \%$ of the participants were unable to swallow at least one of the three sized tablets: of these 13 participants were aged $4-8$ years and one was $9-12$ years. The proportion of tablet naive participants was $77 \%$ in the $4-8$-year-old group and $16 \%$ in the 9-12-year-old group. Seventy-nine per cent of participants who were not able to swallow at least one of the tablets were tablet naive and these were all in the younger age group. These preliminary data suggest that there may be an association between tablet naivety and the ability to swallow a tablet.

As reported in previous studies, the lack of a standardised methodology for the testing of acceptability of paediatric medicines is a barrier to testing acceptability of children's medicines. The methods used in this study are those that have been used in previous acceptability studies, combining patient-reported outcomes and questionnaires with hedonic scales and visual observations, but there is no evidence to demonstrate the validity of these measures. The validity of each participant rating each attribute of the tablets in this study could be questionable, as it is unknown if children as young as 4 years old were able to understand the meaning of each question. It is possible that the interpretation of different questions may have been inconsistent between participants and between age groups. If a larger scale follow-up study is to be carried out, amending the wording of some of the questions on the participant questionnaire would be favourable as there were queries regarding the meaning of the word 'texture' despite explanation by the research team. In addition, some of the younger participants who drank a very large volume of water to enable them to swallow the tablet then went on to rate the volume of water that they were required to consume in order to swallow the tablet as 1 (very good) on the hedonic scale. Therefore, the ratings given by the children were possibly influenced by if they liked drinking water or not, rather than the volume of water required in the context of swallowing the tablet.

The results of the screening data demonstrate that it is extremely difficult to recruit inpatients in the NHS setting to studies testing the acceptability of medicines in children. Of the 250 individuals who were screened for the study on the general medical ward across a 4-week period, only 39 (16\%) children were eligible to approach. Children were ineligible to participate due to a large number of reasons, the most common being that they were under 4 years old. Some other children admitted to the ward were too old and others had infections, complex health issues or disability. Of the 39 eligible inpatients approached by the research team, only 2 were recruited (5\%). Reasons for non-participation of those approached were health issues/disability, refusal, infection, safeguarding issues, patient is off the ward or has been discharged and nut/gluten/lactose allergies. 
Therefore, recruiting on inpatient wards has not proven to be a feasible method of recruitment. It was very difficult to get the parents of sick children to engage in the study as they were solely focused on their child's health and recovery, and did not want another factor to think about. The recruitment of children of staff has proven to be a good method of recruitment, and regulatory permissions are in place to deliver the study outside of the healthcare environment, targeting public events and youth groups such as Brownies and Guides. The study showed that participant fidelity and compliance with the interventions was high. There are other examples in the literature of children being successfully recruited to swallowability/acceptability studies in the inpatient setting for example ${ }^{714}$ with high volumes of participants being recruited; however, a key difference is there were no competing studies compared with the hospital in the study reported here which has over 250 studies running at any one time. It is unlikely that the low recruitment rate observed in the regional hospital is attributable to inadequate institutional capabilities, as over 49000 CYP have been recruited to clinical research studies since 2008. Valuable lessons have been learnt from this feasibility study and it will help to inform the design of a larger definitive trial where the tablet size and administration would be randomised instead of being given by increasing size. The assumption was that the administration of small to large tablet size was the best approach for the younger age group, particularly participants aged 4-6years, who most likely had no previous experience of swallowing tablets. There were also some concerns over the risk of choking. However, this was not found to be the case and when asked if they would be willing to participate in a future study if the tablet size was randomised, $70 \%$ of children would be willing to take part in a randomised study. A larger, randomised controlled trial would overcome the issue of the younger group of participants aged 4 years who had difficulty swallowing the $6 \mathrm{~mm}$ tablet but later went on to swallow the larger sized tablets.

\section{Author affiliations}

${ }^{1}$ Paediatric Medicines Research Unit, Alder Hey Children's NHS Foundation Trust, Liverpool, UK

${ }^{2}$ NIHR Alder Hey Clinical Research Facility, Alder Hey Children's NHS Foundation Trust, Liverpool, UK

${ }^{3}$ Cancer Research UK Clinical Trials Unit, Institute of Cancer and Genomic Sciences, University of Birmingham, Birmingham, UK

${ }^{4}$ Pediatric Services, Quotient Sciences, Nottingham, UK

${ }^{5}$ Department of Clinical and Pharmaceutical Sciences, University of Hertfordshire, Hatfield, UK

Acknowledgements The authors would like to thank Dr Christian DeGoede, Alison Swan and Andrew Lancaster at the National Institute for Health Research (NIHR) Lancashire Clinical Research Facility, Royal Preston Hospital for their contribution to recruitment of patients. All patients, children, families and researchers at the participating sites are acknowledged for their participation in the study. We would also like to thank the staff and participants of the Liverpool YPAG for their valuable contribution to the design of this study. This is a summary of independent research which received no additional funding and was carried out at the NIHR, Alder Hey
Clinical Research Facility. The views expressed are those of the author(s) and not necessarily those of the NHS, the NIHR or the Department of Health.

Contributors MP, LB, FL and PM planned the study, were involved in study design, protocol development and devised the questionnaires. UO, $\mathrm{HJ}$ and NK contributed to the protocol development. LB, PM, SA, EM, JS and FW collected the data. MP, LB and EM analysed and interpreted the data. LB, EM and MP drafted the manuscript. All authors critically revised the manuscript. EM and LB revised the manuscript and all authors read and approved the final manuscript.

Funding The authors have not declared a specific grant for this research from any funding agency in the public, commercial or not-for-profit sectors.

Competing interests None declared.

Patient consent for publication Not required.

Ethics approval Ethical approval for this study was obtained from an NHS Research Ethics Committee (REC), REC Reference 17/NW/0410, and the Health Research Authority, IRAS No. 228 063. The Medicines and Healthcare products Regulatory Agency (MHRA) confirmed it was not required to issue a MHRA Clinical Trials Authorisation as the study only involved placebo tablets.

Provenance and peer review Not commissioned; externally peer reviewed.

Data availability statement Data are available upon reasonable request. All data relevant to the study are included in the article. The full data set is held by the corresponding author, please email with any requests for extra data.

Open access This is an open access article distributed in accordance with the Creative Commons Attribution Non Commercial (CC BY-NC 4.0) license, which permits others to distribute, remix, adapt, build upon this work non-commercially, and license their derivative works on different terms, provided the original work is properly cited, appropriate credit is given, any changes made indicated, and the use is non-commercial. See: http://creativecommons.org/licenses/by-nc/4.0/.

ORCID iD

Louise Bracken http://orcid.org/0000-0002-9632-2252

\section{REFERENCES}

1 Van Riet-Nales DA, Kozarewicz P, Aylward B, et al. Paediatric drug development and formulation Design-a European perspective. AAPS PharmSciTech 2017;18:241-9.

2 European Medicines Agency. Guideline on pharmaceutical development of medicines for paediatric use, 2013. Available: http:// www.ema.europa.eu/docs/en_GB/document_library/Scientific guideline/2013/07/WC500147002.pdf [Accessed 28 Nov 2019].

3 Mistry P, Batchelor H, SPaeDD-UK project (Smart Paediatric Drug Development - UK). Evidence of acceptability of oral paediatric medicines: a review. J Pharm Pharmacol 2017;69:361-76.

4 Mistry P, Batchelor H, SPaeDD-UK project. Methodology used to assess acceptability of oral pediatric medicines: a systematic literature search and narrative review. Paediatr Drugs 2017;19:223-33.

5 Mistry $\mathrm{P}$, Stirling $\mathrm{H}$, Callens $\mathrm{C}$, et al. Evaluation of patient-reported outcome measurements as a reliable tool to measure acceptability of the taste of paediatric medicines in an inpatient paediatric population. BMJ Open 2018;8:e021961.

6 Lajoinie A, Henin E, Nguyen KA, et al. Oral drug dosage forms administered to hospitalized children: analysis of 117,665 oral administrations in a French paediatric Hospital over a 1-year period. Int J Pharm 2016;500:336-44.

7 Klingmann V, Linderskamp $\mathrm{H}$, Meissner $\mathrm{T}$, et al. Acceptability of multiple uncoated Minitablets in infants and toddlers: a randomized controlled trial. J Pediatr 2018;201:202-7.

8 van Riet-Nales DA, de Neef BJ, Schobben AFAM, et al. Acceptability of different oral formulations in infants and preschool children. Arch Dis Child 2013;98:725-31.

9 Tse Y, Vasey N, Dua D, et al. The KidzMed project: teaching children to swallow tablet medication. Arch Dis Child 2019. doi:10.1136/ archdischild-2019-317512. [Epub ahead of print: 08 Oct 2019].

10 Salunke S, Liu F, Batchelor H, et al. European Paediatric Formulation Initiative (EuPFI)-Formulating Ideas for Better Medicines for Children. AAPS PharmSciTech 2017;18:257-62.

11 Ternik R, Liu F, Bartlett JA, et al. Assessment of swallowability and palatability of oral dosage forms in children: report from an M-CERSI pediatric formulation workshop. Int J Pharm 2018;536:570-81.

12 Arain M, Campbell MJ, Cooper CL, et al. What is a pilot or feasibility study? A review of current practice and editorial policy. BMC Med Res Methodol 2010;10:67. 
13 European Medicines Agency. Reflection paper on the pharmaceutical development of medicines for use in the older population (draft). Available: https://www.ema.europa.eu/en/documents/scientificguideline/reflection-paper-pharmaceutical-development-medicinesuse-older-population-first-version_en.pdf [Accessed 15 Apr 2020]

14 Klingmann V, Spomer N, Lerch C, et al. Favorable acceptance of mini-tablets compared with syrup: a randomized controlled trial in infants and preschool children. J Pediatr 2013;163:1728-32.
15 Beck MH, Cataldo M, Slifer KJ, et al. Teaching children with attention deficit hyperactivity disorder (ADHD) and autistic disorder (AD) how to swallow pills. Clin Pediatr 2005;44:515-26.

16 Hansen DL, Tulinius D, Hansen EH. Adolescents' struggles with swallowing tablets: barriers, strategies and learning. Pharm World Sci 2008;30:65-9.

17 Funk MJ, Mullins LL, Olson RA. Teaching children to swallow pills: a case study. Children's Health Care 1984;13:20-3. 